\title{
Ex vivo liver resection for tumoral pathology - benefits, risks and outcomes
}

\author{
Nicolae Bacalbasa ${ }^{1,2}$, Irina Balescu3 ${ }^{3}$, Adina Croitoru ${ }^{4,5}$, Simona Dima ${ }^{4}$, \\ Ana Maria Bianca Mitricof ${ }^{5}$, Mihaela Vilcu ${ }^{1,2}$, Iulian Brezean ${ }^{1,2}$ \\ 1"Carol Davila" University of Medicine and Pharmacy, Bucharest, Romania \\ 2"Ion Cantacuzino" Clinical Hospital, Bucharest, Romania \\ 3"Ponderas" Academic Hospital, Bucharest, Romania \\ ${ }^{4}$ Fundeni Clinical Institute, Bucharest, Romania \\ ${ }^{5}$ Faculty of Medicine, "Titu Maiorescu" University, Bucharest, Romania
}

\begin{abstract}
Ex vivo liver surgery techniques have been proposed almost two decades ago in order to extend the indications and feasibility of liver resections for large, but not absolutely unresectable liver lesions. However, the procedure remains a demanding one, being associated with high risks of postoperative complications; therefore it should be performed only in specialized centers. This is a literature review of the largest studies conducted on the theme of ex vivo liver resection for tumoral pathology.
\end{abstract}

Keywords: ex vivo resection, autotransplantation, liver tumors

\section{INTRODUCTION}

Proposed for the first time by James Hrdy in 1963 in order to treat an ureteral injury, the concept of ex vivo surgery consists of explanting a pathological organ, reconstruct it outside of the body by the means of cold preservation and reimplantation of the unaffected part of the organ (1). This principle was applied for the first time in order to resect a conventionally unresectable liver tumor by Pichlmayr et al. in 1988 (2). At that moment the German authors performed an ex vivo resection for hepatic metastases originating from a leiomyosarcoma in a 40 -years-old patient. The patient's liver was explanted, maintained in a cardioplegic (histidinetryptophan-ketoglutarate) solution, the lesions were resected and the remnant liver parenchyma was implanted six hours later; during the anhepatic period the inferior cava vein as well as the portal vein were decompressed by using an extracorporeal femoro-porto-axillary bypass (2).

\section{THE PRINCIPLE OF THE METHOD}

The method of ex vivo liver resections consists of completely removing the liver from the patient, in this time the hepatic perfusion being provided by the administration of a solution with preservation functions. In these conditions a bloodless transection of the liver parenchyma is performed, the tumoral masses are resected and, if necessary, the vascular structures such as portal vein or hepatic veins are reconstructed; once this phase is completed, the remnant liver is reimplanted (3). The method can provide in this way the radical resection of complex lesions which could not be approached in other manners such as performing a Pringle manoeuvre. The main difference between the two methods is the type of ischemia and the length of ischemia. Therefore, if Pringle manoeuvre is performed, a warm ischemia is induced and it should not exceed one hour (in extreme cases 90 minutes have been proposed if the liver parenchyma is a 
normal, non-cirrhotic one); however, this interval might be too short in order to perform complex liver resection. In consequence, in such cases cold ischemia and protective hypothermia have been proposed with encouraging results. The method can be performed under three aspects: in situ resection - in this case the liver is perfused with hypothermic solutions and the major vessels are cross-clamped, ante-situm resection - the suprahepatic part of the cava vein is dissected and ex-situ resection when the liver is completely removed from the body (4).

The procedure of ex-vivo resections begins with the exploration of the abdominal cavity in order to rule out the existence of extrahepatic tumors and with the complete mobilization of the liver and of the retrohepatic segmet of the cava vein. During the anhepatic period the circulation is provided by performing a bypass from the portal vein and the left femoral vein to the left axillary vein; the hypothermic perfusion phase is initiated only after the bypass is functioning and uses the same solutions as in transplantation (such as histidine- tryptophanketoglutarate $=$ HTK $=$ Custodiol). Once the specimen is explanted, the resectional phase begins and consists of dissecting and removing all the suspect lesions followed by suturing and reconstructing the transsected vascular structures and implanting the remnant liver; when it comes to the reconstruction of the resected vascular structures, autologous segments of the saphenous vein or alloplastic prosthesis such as polytetrafluororethylene might be used (4).

\section{INDICATIONS OF EX VIVO LIVER SURGERY FOR TUMORAL PATHOLOGY}

The method has been used in the last decades in order to treat benign pathologies such as liver echinococcosis or different types of malignant tumors (5-7). Therefore the method provided the possibility to resect extended, otherwise considered as unresectable liver lesions as well as pancreatic head tumors invading the hepatic hilum or soft tissue tumors involving the hepatoduodenal ligament and compressing the surrounding viscera $(8,9)$. The best candidates for this type of surgery are represented by the cases presenting a benign tumoral pathology or a low grade malignant tumor which is amenable to $\mathrm{R} 0$ resection and in whom a long term survival is expected (8). As for the reason of which the lesion is considered as unresectable, it should be related to the local extent of the tumoral process and not to the presence of extrahepatic liver metastases (8).

\section{BENEFITS OF EX VIVO LIVER SURGERY FOR TUMORAL PATHOLOGY}

The method of ex vivo liver surgery provides the benefits of resection of otherwise unresectable liver lesions, offering in this way the benefits of an $\mathrm{R} 0$ resection for various types of malignant tumors and increasing in this way the chance for cure. Moreover, it minimizes the risks of ischemic injury by the use of cold preservation (8).

Another benefit of ex vivo liver surgery is the one that it can offer a chance for cure for patients who could be otherwise treated only if liver transplantation could be performed; therefore, in these conditions there is no need to search for a deceased or a living donor in a society in which the number of donors still remains extremely low (8). In the meantime the patient in whom an ex vivo liver resection is performed won't need any postoperative immunosuppressive treatment (8). One of the first studies which came to demonstrate the effectiveness of the method for tumoral pathology comes from Hanover, Germany, the place where the first such procedure was performed. This study was conducted on a period of ten years and included 54 surgeries performed in 52 cases, the most commonly encountered indications being represented by colorectal liver metastases (in 21 cases), liver metastases from other primaries (in 12 cases), primary hepatobiliopancreatic tumors (in14 cases) and other pathologies (in five cases). The postoperative morbidity and mortality rate was of $30 \%$, the most commonly encountered issue being related to the development of liver failure. As for the long term outcomes, the best results were reported by the cases submitted to surgery for colorectal liver metastases, the median survival time being of 21 months. However, the most important problem which was reported when it came to the long term outcomes was the one regarding the development of recurrent disease (4).

Another interesting series of patients submitted lo ex vivo liver surgery was published by Oldhafer et al. in 2000 and included 24 patients with various primary and secondary liver tumors (in 22 cases) as well as with benign disease (in two cases) (10). The procedure was initially successful in 22 cases, in the other two cases auto-transplantation being not feasible; the anhepatic period was of $5.6+/-1$, 1 hours. Among the 24 cases there were four patients who developed liver failure after autotransplantation and required retransplantation. Among the 24 cases, 15 cases survived and were discharged after $36,5+/-16$ days; as for the long term outcomes, cas- 
es submitted to surgery for colorectal liver metastases reported a median survival of 21 months while patients submitted to surgery for benign tumors were still alive at nine and five years respectively after surgery (10).

A study which included solely the patients submitted to ex vivo resections for colorectal liver metastases was conducted by Lodge et al. and was published in 2000 (11). The study included eight patients submitted to surgery between 1995-1999, four cases presenting a single metastasis, three other cases presenting two lesions while the eight case presented three lesions; moreover, in all cases segmental resection of the inferior cava vein was needed while the reconstruction was performed by using autologous materials or allografts; postoperatively, two patients died within the first month. The other six cases were alive at a follow-up period of five to 12 months (11).

The method has been reported to have encouraging results also in selected cases of patients diagnosed with otherwise unresectable hepatocarcinomas. In the study published by Wen et al. in 2013 the authors reported a series of three such cases submitted to surgery in 2011 in Changhua Hospital, Taiwan (12). In all cases the procedure was successfully performed by using a portocaval shunt instead of a veno-venous bypass in order to increase the hemodynamic stability; the remnant liver volume was of $44.26 \%, 34.46 \%$ and $51 \%$ respectively, all patients being discharged in a good clinical status. When it comes to the long term outcomes, the authors reported that all cases were still alive when conducting the study (at 28 months, 26 months and 23 months respectively after surgery), two of the three patients being diagnosed and successfully treated for recurrent disease eight months after the ex vivo surgery (12).

In order to improve the outcomes of these patients and to provide a less invasive manner of treating such cases, the Chinese authors proposed the first models of laparoscopic training systems which are used for the moment in porcine models with promising results (13).

\section{PERIOPERATIVE RISKS OF EX VIVO LIVER SURGERY FOR TUMORAL PATHOLOGY}

However, the method is an extremely demanding one and associates increased risks of perioperative complications such as vascular thrombosis (8). Moreover, the method seems to be associated with the development of cholestasis syndrome in certain cases due to the presence of a period of ischemia, therefore, it should not be performed in cases in which preoperative cholestasis syndrome is present (14).

Another very important issue which should be taken in consideration after performing this type of surgery is related to the remnant liver volume; the Chinese authors conducted by Shen et al. investigated whether a remnant liver volume lower than $40 \%$ of the estimated standard liver volume could associate any postoperative risks after ex vivo surgery; surprisingly, the authors revealed the fact that a lower than $40 \%$ ratio is not associated with an increased risk of postoperative complications (15).

\section{COMBINING THE TECHNIQUE OF EX VIVO LIVER RESECTIONS WITH OTHER LIVER SURGERY TECHNIQUES}

In order to maximize the benefits of various techniques of liver surgery and to achieve the resectability of tumoral lesions, certain authors proposed the combination of ex vivo liver resection with other pre-existing techniques such as liver partition and portal vein ligation with encouraging results. Therefore, Baimas-George et al. recently reported the case of a 73-year-old patient diagnosed with a large cholangiocarcinoma invading the inferior cava vein, the three hepatic veins and the right anterior portal pedicle in whom liver partition and portal vein ligation followed by ex vivo resection of the remnant liver tumor was performed, an R0 resection being achieved (16).

Another successful report comes from Ian McGilvray et al. who presented the case of a 43-year-old patient who was diagnosed with a large leiomyosarcoma of the inferior cava vein which extended to the right atrium, to the right hepatic vein and to the right kidney. Intraoperatively thrombosis of the middle and left hepatic veins was seen, an acute Budd Chiari syndrome being developed, so the patient was submitted to an ex vivo liver resection of the segments I, VI, VII in association with right nephrectomy, right adrenalectomy, atrio-caval resection and vascular reconstruction by using a polytetrafluoroethylene grafts the early and middle term results were favourable, the patient being alive and free of recurrence eight months later (17).

\section{CONCLUSIONS}

Ex vivo liver resections for tumoral pathology represent demanding surgical procedures which 
should be performed in high volume centers with expertise in liver transplantation and should be reserved for selected cases. The procedure is associated with significant risks of postoperative complications such as extended vascular thrombosis but it offers a chance for cure in cases presenting large, practically unresectable lesions by the means of the standard resection techniques.

\section{REFERENCES}

1. HARDY JD. High ureteral injuries. Management by autotransplantation of the kidney. JAMA 1963; 184: 97-101.

2. Pichlmayr R, Bretschneider HJ, Kirchner E et al. [Ex situ operation on the liver. A new possibility in liver surgery]. Langenbecks Arch.Chir 1988; 373: 122-6.

3. Hemming AW, Chari RS, Cattral MS. Ex vivo liver resection. Can.J.Surg. 2000; 43: 222-4.

4. Raab R, Schlitt HJ, Oldhafer $\mathrm{KJ}$ et al. Ex-vivo resection techniques in tissue-preserving surgery for liver malignancies. Langenbecks Arch. Surg. 2000; 385: 179-84.

5. Wen $\mathrm{H}$, Dong JH, Zhang JH et al. Ex Vivo Liver Resection and Autotransplantation for End-Stage Alveolar Echinococcosis: A Case Series. Am.J.Transplant. 2016; 16: 615-24.

6. Koch S, Bresson-Hadni S, Miguet JP et al. Experience of liver transplantation for incurable alveolar echinococcosis: a 45-case European collaborative report. Transplantation 2003; 75: 856-63.

7. Wen $\mathrm{H}$, Dong $\mathrm{JH}$, Zhang $\mathrm{JH}$ et al. Ex vivo liver resection followed by autotransplantation for end-stage hepatic alveolar echinococcosis. Chin Med.J.(Engl.) 2011; 124: 2813-7.

8. Hwang R, Liou P, Kato T. Ex vivo liver resection and autotransplantation: An emerging option in selected indications. J.Hepatol. 2018; 69: 1002-3.

9. Matsuoka N, Weiner JI, Griesemer AD et al. Ex vivo pancreaticoduodenectomy and liver autotransplantation for pancreatic head tumor with extensive involvement of the hepatoduodenal ligament. Liver Transpl. 2015; 21: 1553-6.

\section{Acknowledgement}

This work was supported by the project entitled „Multidisciplinary Consortium for Supporting the Research Skills in Diagnosing, Treating and Identifying Predictive Factors of Malignant Gynecologic Disorders", project number PN-III-P1-1.2-PCCDI2017-0833.
10. Oldhafer $\mathrm{KJ}$, Lang $\mathrm{H}$, Schlitt $\mathrm{HJ}$ et al. Long-term experience after ex situ liver surgery. Surgery 2000; 127: 520-7.

11. Lodge JP, Ammori BJ, Prasad KR et al. Ex vivo and in situ resection of inferior vena cava with hepatectomy for colorectal metastases. Ann. Surg. 2000; 231: 471-9.

12. Wen $\mathrm{PH}$, Lin $\mathrm{KH}$, Chen $\mathrm{YL}$ et al. Extracorporeal hepatic resection and autotransplantation using temporary portocaval shunt provides an improved solution for conventionally unresectable HCC. Dig.Dis.Sci. 2013; 58: 3637-40.

13. Liu W, Zheng X, Wu R et al. Novel laparoscopic training system with continuously perfused ex-vivo porcine liver for hepatobiliary surgery. Surg.Endosc. 2018; 32: 743-50.

14. Pichlmayr R, Grosse H, Hauss J et al. Technique and preliminary results of extracorporeal liver surgery (bench procedure) and of surgery on the in situ perfused liver. Br.J.Surg. 1990; 77: 21-6.

15. Shen S, Qiu Y, Yang X et al . Remnant Liver-to-Standard Liver Volume Ratio Below $40 \%$ is Safe in Ex Vivo Liver Resection and Autotransplantation. J.Gastrointest.Surg. 2018.

16. Baimas-George MR, Levi DM, Eskind LB et al. Ex vivo liver resection coupled with associated liver partition and portal vein ligation: Combining existing techniques to achieve surgical resectability. J.Surg. Oncol. 2019; 119: 771-6.

17. Ex-vivo liver I/VI/VII segmentectomy with en bloc caval resection and right nephrectomy - Presented by Dr. Ian McGilvray. Toronto Video Atlas of Surgery. 